\title{
Utility and Limitations of Glycated Hemoglobin (HbA1c) in Patients with Liver Cirrhosis as Compared with Oral Glucose Tolerance Test for Diagnosis of Diabetes
}

\author{
Tejasav Sehrawat · Anuraag Jindal · Paaras Kohli · Amit Thour • \\ Jasbinder Kaur · Atul Sachdev • Yashdeep Gupta
}

Received: December 5, 2017 / Published online: January 5, 2018

(C) The Author(s) 2018. This article is an open access publication

\section{ABSTRACT}

Introduction: To study the utility of glycated hemoglobin (HbA1c) in the diagnosis of diabetes in patients with cirrhosis as compared to the gold standard oral glucose tolerance test (OGTT) and to see the effect of anemia and severity of cirrhosis on its performance.

Methods: Individuals $(n=100)$ with an established diagnosis of liver cirrhosis were recruited. The OGTT was performed as described by the World Health Organization (WHO). The

Enhanced content To view enhanced content for this article go to http://www.medengine.com/Redeem/ 3E1DF060406A1CCC.

At the time of carrying out this study, A. Jindal and Y. Gupta were affiliated to Department of Internal Medicine, Government Medical College and Hospital, Chandigarh, India.

T. Sehrawat · P. Kohli · A. Thour · A. Sachdev Department of Internal Medicine, Government Medical College and Hospital, Chandigarh, India

A. Jindal

Department of Gastroenterology, Columbia Asia Hospital, Patiala, India

J. Kaur

Department of Biochemistry, Government Medical College and Hospital, Chandigarh, India

\section{Y. Gupta $(\bowtie)$}

Department of Endocrinology and Metabolism, All India Institute of Medical Sciences, New Delhi, India e-mail: yash_deep_gupta@yahoo.co.in severity of cirrhosis was calculated using the Child-Turcotte-Pugh (CTP) score. The severity of anemia was defined according to WHO criteria. The utility of HbA1c was compared against the OGTT results. Test sensitivity and specificity were used to describe the diagnostic accuracy of HbA1c.

Results: A total of 100 subjects aged $46.9 \pm 9.1$ years (mean \pm standard deviation) participated in the study, of whom $65 \%$ were recruited from out patient department of our hospital. The overall sensitivity and specificity of a HbA1c level of $\geq 6.5 \%$ for the diagnosis of diabetes in patients with cirrhosis was $77.1 \%$ (95\% CI 59.9, 89.6) and 90.8\% (95\% CI 81.0, 96.5), respectively. The positive and negative predictive values were $81.8 \%$ (95\% CI 67.3, 90.8) and $88.1 \%$ (95\% CI 80.0, 93.2), respectively. The area under the curve was 0.85 (95\% CI 0.75-0.94). The sensitivity of HbA1c for diagnosing diabetes in outpatients was $87.0 \%$ $(95 \%$ CI $66.4,97.2)$ and was better than that for diagnosing diabetes in hospitalized patients (58.3\%; 95\% CI 27.7, 84.8). The sensitivity of HbA1c for diagnosing diabetes was poor in patients with moderate to severe anemia. The difference in sensitivity and specificity was not statistically different for CTP classes A, B and C. The prevalence of diabetes as defined by American Diabetes Association OGTT criteria was 35\% (95\% CI 25.7-45.2\%).

Conclusions: Taking OGTT as the gold standard, the sensitivity of HbA1c for diagnosing 
diabetes is good when used in outpatients with cirrhosis. However, the sensitivity of HbA1c decreases when it is used for hospitalized patients, suggesting that it is not a good test for diagnosis of diabetes in such cases. It also performs poorly if the patient has moderate to severe anemia.

Keywords: Hepatogenous diabetes; HbA1c; OGTT; Cirrhosis; Diagnosis

\section{INTRODUCTION}

Liver cirrhosis is a pathologically defined disease [1]. Histologically it is characterized by diffuse nodular regeneration surrounded by dense fibrotic septa. Subsequent parenchymal extinction and collapse of liver structures together cause pronounced distortion of hepatic vascular architecture [2]. Liver biopsy is the gold standard, but it is not been widely used in clinical practice as it is invasive and susceptible to sampling error and inter-observer discrepancy. Clinical diagnosis is preferentially made on the basis of patient history, physical examination, laboratory and imaging findings. In cases where there is discrepancy among the findings, liver biopsy is helpful for a definitive diagnosis of cirrhosis [1]. Hepatitis C virus, alcohol misuse, non-alcoholic fatty liver disease are the main causes of cirrhosis in developed countries, whereas infection with hepatitis B virus is the most common cause in sub-Saharan Africa and most parts of Asia [2].

Cirrhosis is the fourteenth most common cause of death worldwide and results in approximate 1.03 million deaths per year worldwide [3]. Diabetes is an independent factor for poor prognosis in patients with cirrhosis. Specifically, diabetes is associated with the occurrence of major complications of cirrhosis, including ascites, renal dysfunction, hepatic encephalopathy and bacterial infections. Diabetes is also associated with an increased risk of hepatocellular carcinoma and mortality in patients with chronic liver diseases [4].

Diabetes is a disorder of chronic hyperglycemia and has traditionally been subdivided into type 1 diabetes (with autoimmune destruction of insulin-secreting $\beta$ cells) and type 2 diabetes (T2DM; with insulin resistance and features of metabolic syndrome) [5]. The fasting plasma glucose (FPG) test, $75 \mathrm{~g}$ oral glucose tolerance test (OGTT), measurement of random plasma glucose or glycated hemoglobin (HbA1c) values are four methods used to diagnose diabetes [6]. The global epidemic of diabetes mellitus and its complications pose a major health threat worldwide [7]. Diabetes mellitus is the ninth major cause of death [8]. The International Diabetes Federation (IDF) estimated that worldwide one in 11 adults aged 20-79 years (415 million adults) had diabetes mellitus in 2015. This estimate is projected to rise to 642 million by 2040. China and India are the top two epicenters [9]. Genetic predisposition partly determines individual susceptibility to T2DM, but an unhealthy diet and a sedentary lifestyle are important drivers of the current global epidemic. Early developmental factors (such as intrauterine exposures) also have a role in susceptibility to T2DM later in life [7].

Disorders of glucose metabolism, namely glucose intolerance and diabetes, are frequent in patients with chronic liver diseases. The prevalence of diabetes is higher in patients with cirrhosis than in those without cirrhosis [10]. In patients with cirrhosis, disorders of glucose metabolism range from mere glucose intolerance to overt diabetes. It is estimated that only $30 \%$ of these patients have normal glucose tolerance, with 30-50\% having impaired glucose tolerance and up to $30 \%$ having overt diabetes. These values are much higher than those in the general population, where the prevalence of glucose intolerance is around $15 \%$ and that of diabetes is $8 \%$. In patients with cirrhosis, diabetes can either be the classical T2DM or the socalled hepatogenous diabetes, i.e. a consequence of liver insufficiency and portal hypertension [4, 10]. Cirrhosis is associated with portosystemic shunts as well as reduced hepatic mass, both of which can impair insulin clearance by the liver, contributing to peripheral insulin resistance through insulin receptor downregulation. Moreover, cirrhosis is associated with increased levels of advanced glycation endproducts and hypoxia-inducible factors, 
both of which may play a role in the development of diabetes [4].

HbA1c measurement is used in the routine evaluation and management of patients with diabetes [6]. In 2016, Nadelson et al. reported that $\mathrm{HbA1c}$ is an unreliable indicator of glycemic control in patients with cirrhosis due to the high prevalence of concomitant anemia in these patients [11]. The data in this study originated from studies performed prior to the adoption of HbA1c as one of the diagnostic criteria for diabetes and suggest that HbA1c values are lower than expected and are not reliable indicators either for diagnosis or the management of diabetes [12-16]. Hence it was recommended that the OGTT be used for diagnosis and that monitoring should be done on the basis of blood glucose or fructosamine values. Orsi et al. [10] reviewed all studies pertaining to diagnosis of diabetes in patients with cirrhosis. These authors found that out of nine studies, only two diagnosed diabetes on the basis of HbA1c measurement, and none compared the efficacy of HbA1c measurement and the OGTT $[17,18]$. Therefore, there remains an important knowledge gap in the available literature in that the performance of the current recommended cutoff of HbA1c (6.5\%) for diagnosis of diabetes has not been evaluated against that of OGTT as the gold standard [4, 11-18].

The aim of this study was to evaluate the utility of HbA1c measurement as compared to the OGTT for the diagnosis of diabetes in patients with cirrhosis. We also studied the effect of anemia and severity of liver disease on the diagnostic utility of HbA1c and separately analyzed the results for outpatients and those admitted to the hospital. Apart from the prevailing knowledge gap in literature, the rationale behind the study was that all cirrhotic patients may not behave in the same manner. There may be certain subgroups where HbA1c may have optimal sensitivity and specificity for the diagnosis of diabetes, and thus its measurement may serve as an important diagnostic tool. This possibility is all the more important when the fact is considered that all cirrhotic patients may not be in a position to undergo OGTT or may not prefer the OGTT for diagnosis of diabetes.

\section{METHODS}

A "convenient sample" of 100 liver cirrhosis patients were enrolled in this cross-sectional prospective study from the inpatient (IPD) and outpatient departments (OPD) of Internal medicine, Government Medical College and Hospital, Chandigarh, between October 2013 and April 2015. Patients unable to undergo the OGTT or those with a history of gastrointestinal bleed/blood transfusion were excluded, as were patients with known diabetes. The study was approved by ethics committee. All procedures performed were in accordance with the ethical standards of the institutional research committees of the participating hospital and with the 1964 Helsinki declaration and its later amendments or comparable ethical standards. Informed consent was obtained from all individual participants included in the study.

The diagnosis of cirrhosis was made by a gastroenterologist (AS, AJ) on the basis of clinical spectrum, substantiated by ultrasonography of liver (irregular and nodular liver; other findings include small and shrunken liver, splenomegaly) and presence of complications of cirrhosis, including ascites, sepsis, variceal bleeding, encephalopathy and non-obstructive jaundice [2]. The severity of chronic liver disease (CLD) was calculated using Child-Turcotte-Pugh (CTP) scores [19]. The severity of anemia was defined according to World Health Organization (WHO) criteria, including the recommendations for adult men and adult non-pregnant women [20]. The in-patients underwent investigations when in a stable condition before discharge.

The OGTT was performed as described by the WHO guidelines [21]. Blood glucose was analyzed using the glucose oxidase peroxidase method in a Modular P800 analyzer (Roche Diagnostics, Mannheim, Germany) using colorimetric method. HbA1c was measured using the turbid metric inhibition immunoassay, utilizing Roche kits in a Modular P800 analyzer.

Individuals were classified as being normoglycemic (FPG $<5.6 \mathrm{mmol} / \mathrm{L}$ and 2 -h plasma glucose $<7.8 \mathrm{mmol} / \mathrm{L}$ ), having prediabetes (FPG $=5.6-6.9 \mathrm{mmol} / \mathrm{L}$ and/or 2 -h plasma glucose $=7.8-11 \mathrm{mmol} / \mathrm{L}$ ) or having diabetes (FPG 
$\geq 7 \mathrm{mmol} / \mathrm{L}$ and/or 2-h plasma glucose $\geq 11.1 \mathrm{mmol} / \mathrm{L}$ ) [6]. The performance of the HbA1c measurement was assessed against OGTT criteria for diagnosis of diabetes.

Statistical analysis was carried out using STATA 12.0 (StataCorp LP, College Station, TX). Data were presented as the number with percentage or as the mean \pm standard deviation (SD) with the median and range, as appropriate. Test sensitivity and specificity were used to describe the diagnostic accuracy of HbA1c. Receiver operating characteristic curves were generated to determine the optimal sensitivity and specificity, and the area under the curves (AUC) were compared using the Chi-square test. Significance levels were set at $p<0.05$.

\section{RESULTS}

A total of 100 subjects with an established diagnosis of liver cirrhosis participated in the study, of whom $65 \%$ were recruited from the OPD. The mean ( \pm SD) age was 46.9 \pm 9.1 years, men accounted for $90 \%$ of the subjects and $90 \%$ of the subjects were anemic. The mean hemoglobin level was $10.3 \pm 2.2 \mathrm{~g} / \mathrm{L}$. Regarding the CTP class, $22.7 \%$ were in CTP class A and $35.1 \%$ were in CTP class C. The prevalence of diabetes was $35 \%$ [95\% CI 25.7-45.2\%]; 23\% were normoglycemic and $42 \%$ had prediabetes based on the OGTT results. The etiology for $80.2 \%$ of patients with cirrhosis was alcohol, with hepatitis B virus responsible for $5.9 \%$ of cirrhosis cases, hepatitis $\mathrm{C}$ virus for $4.0 \%$, alcohol and hepatitis B for $6.9 \%$, alcohol and hepatitis $\mathrm{C}$ for $2.0 \%$ and combined hepatitis $\mathrm{B}$ and $\mathrm{C}$ infection for $1.0 \%$ of cases. In terms of comorbidities, $69 \%$ of subjects had ascites, $19 \%$ had spontaneous bacterial peritonitis, 32\% had esophageal-gastric varices and $19 \%$ had a history of hepatic encephalopathy. Of the 35 patients diagnosed with diabetes, five had isolated abnormalities in the FPG test, nine were detected only with the $2 \mathrm{~h}$ value on the OGTT test and $21 \mathrm{had}$ abnormal values for both tests. The important characteristics of patients with and without diabetes are presented in Table 1.

Table 1 Baseline characteristics of study patients

\begin{tabular}{llll}
\hline Parameter & Total $(\boldsymbol{N}=\mathbf{1 0 0})$ & Diabetes $(\boldsymbol{n}=35)$ & No diabetes $(\boldsymbol{n}=\mathbf{6 5})$ \\
\hline Age (mean \pm SD) & $46.9 \pm 9.1$ & $47.3 \pm 9.3$ & $46.6 \pm 9$ \\
Male sex (\%) & 90 & 85.7 & 92.3 \\
Recruited through out-patient department (\%) & 65 & 65.7 & 64.6 \\
No or mild anemia (\%) & 42 & 48.6 & 38.5 \\
Moderate or severe anemia (\%) & 58 & 51.4 & 61.5 \\
Hemoglobin (mean \pm SD) & $10.3 \pm 2.2$ & $10.4 \pm 2.3$ & $10.3 \pm 2.2$ \\
CTP class A score (\%) & 22.7 & 29.4 & 19.1 \\
CTP class B score (\%) & 42.3 & 29.4 & 49.2 \\
CTP class C score (\%) & 35.1 & 41.2 & 31.7 \\
Fasting plasma glucose (mmol/L) & $5.9(3.3-16.1)$ & $7.9(4.8-16.1)$ & $5.4(3.3-6.8)$ \\
2 h post glucose plasma value (mmol/L) & $8.9(5.6-27.2)$ & $13.3(8.1-27.2)$ & $7.8(5.6-10.3)$ \\
HbAlc (\%) & $5.9(4-11.1)$ & $7.1(4.2-11.1)$ & $5.3(4-8.6)$ \\
HbAlc (mmol/mol) & $41(20-98)$ & $54.1(22-98)$ & $34.4(20-70)$ \\
\hline
\end{tabular}

Values in parenthesis represents median (range)

SD, Standard deviation; Child-Turcotte-Pugh, Child-Turcotte-Pugh; HbAlc, glycated hemoglobin 


\section{Utility of HbA1C in the Diagnosis of Diabetes as Compared to OGTT}

The overall sensitivity and specificity of HbA1c $\geq 6.5 \%$ for the diagnosis of diabetes in cirrhotic patients was $77.1 \%(95 \%$ CI $59.9,89.6)$ and $90.8 \%$ (95\% CI 81.0, 96.5), respectively. The positive and negative predictive values were $81.8 \%$ (95\% CI $67.3,90.8)$ and $88.1 \%$ (95\% CI 80.0, 93.2), respectively. The AUC was 0.85 (95\% CI 0.75-0.94).

\section{Utility of HbA1C in the Diagnosis of Diabetes in Outpatient and Inpatient Settings}

The sensitivity of HbA1c for the diagnosis of diabetes in outpatients was $87.0 \%$ (95\% CI 66.4, 97.2) and was better than that for hospitalized ones $(58.3 \%$; 95\% CI 27.7, 84.8). The corresponding values for specificity were $92.9 \%(95 \%$ CI $80.5,98.5$ ) and $87.0 \%$ (95\% CI 66.4, 97.2), respectively (Table 2). The AUC for outpatient department and hospitalized patients was 0.896 $(95 \% \quad$ CI $0.795-0.995)$ and 0.77 (95\% CI 0.59-0.96), respectively ( $p$ for comparison between two $\mathrm{AUC}=0.26$ ).

\section{Effect of Anemia on Diagnostic Utility of HbA1c for Diabetes}

We further compared the values for individuals with normal hemoglobin levels or with mild anemia to individuals with moderate or severe anemia. The sensitivity of HbA1c was higher in the former group (82.4\%; 95\% CI 56.6-96.2\%) than in the latter group $(72.2 \% ; 46.5,90.3)$. A similar trend was appreciable in specificity as well: 96\% (95\% CI 79.7-99.9\%) in patients with no/mild anemia compared to $86.9 \%(71.9,95.6)$ in patients with moderate/severe anemia (Table 2). The AUC for patients with mild/no and moderate/severe anemia was 0.90 (95\% CI 0.79-1.00) and 0.80 (95\% CI 0.65-0.94), respectively ( $p$ for comparison between two $\mathrm{AUC}=0.27$.

\section{Effect of Severity of Cirrhosis on the Diagnostic Utility of HbA1c for Diabetes}

No trend was noted for sensitivity and specificity for severity of chronic liver disease as depicted in Table 2. The specificity was comparable in all CTP classes. The sensitivity was less in CTP class B than in CTP classes A and C.

Table 2 Sensitivity, specificity, positive and negative predictive value of glycated hemoglobin in the diagnosis of diabetes (overall and subgroup analysis

\begin{tabular}{lllll}
\hline Setting & Sensitivity & Specificity & $\begin{array}{l}\text { Positive predictive } \\
\text { value }\end{array}$ & $\begin{array}{l}\text { Negative predictive } \\
\text { value }\end{array}$ \\
\hline Overall $(N=100)$ & $77.1(59.9,89.6)$ & $90.8(81.0,96.5)$ & $81.8(67.3,90.8)$ & $88.1(80.0,93.2)$ \\
Indoor patients $(n=35)$ & $58.3(27.7,84.8)$ & $87.0(66.4,97.2)$ & $70.0(42.3,88.1)$ & $80.0(66.8,88.9)$ \\
Outdoor patients $(n=65)$ & $87.0(66.4,97.2)$ & $92.9(80.5,98.5)$ & $87.0(68.9,95.3)$ & $92.9(81.9,97.4)$ \\
No and mild Anemia $(n=42)$ & $82.4(56.6,96.2)$ & $96.0(79.7,99.9)$ & $93.3(67.0,98.9)$ & $88.9(74.1,95.7)$ \\
$\begin{array}{l}\text { Moderate and severe anemia } \\
\quad(n=56)\end{array}$ & $72.2(46.5,90.3)$ & $86.9(71.9,95.6)$ & $72.2(52.3,86.1)$ & $86.8(75.6,93.4)$ \\
CTP class A $(n=22)$ & $80.0(44.4,97.5)$ & $91.7(61.5,99.8)$ & $88.9(54.4,98.2)$ & $84.6(61.2,95.1)$ \\
CTP class B $(n=41)$ & $70.0(34.8,93.3)$ & $90.3(74.3,98.0)$ & $70.0(42.5,88.1)$ & $90.3(78.3,96.1)$ \\
CTP class C $(n=34)$ & $78.6(49.2,95.3)$ & $90.0(68.3,98.8)$ & $84.6(59.0,95.5)$ & $85.7(68.5,94.3)$ \\
\hline
\end{tabular}

Values in table are presented as a percentage with the $95 \%$ confidence interval in parenthesis 


\section{DISCUSSION}

The important findings from our study are as follows. The overall sensitivity and specificity of HbA1c $\geq 6.5 \%$ for the diagnosis of diabetes in cirrhotic patients was 77.1\% (95\% CI 59.9, 89.6) and $90.8 \%$ (95\% CI 81.0, 96.5), respectively. The AUC was 0.85 (95\% CI 0.75-0.94). The sensitivity of HbA1c for the diagnosis of diabetes in outpatients was $87.0 \%$ (95\% CI 66.4, 97.2) and was better than that in hospitalized patients (58.3\%; 95\% CI 27.7, 84.8). The sensitivity of HbA1c was poor in patients with moderate to severe anemia. The difference in sensitivity and specificity was not statistically different for CTP classes A, B and C. The prevalence of diabetes as defined by the American Diabetes Association OGTT criteria was $35 \%$ (95\% CI 25.7-45.2\%).

The prevalence of diabetes in our study was $35 \%$, which is comparable to the prevalence reported in other studies, namely, 6.3- 57.2\%, with the majority of studies reporting a prevalence of between 32 and $37.5 \%[10,11]$. The general prevalence of diabetes in the region is $13.6 \%$ [22].

HbA1c in diabetes mellitus is considered to be a reliable test to assess chronic glycemia and is recommended for both the diagnosis and monitoring of the disease. However, it is considered to be neither accurate nor reliable in patients with cirrhosis due to the various kinds of anemia common in liver disease [23]. Cacciatore et al. used the OGTT on patients with cirrhosis and found that $27 \%$ had diabetes [12]. However, these authors also found that HbA1c levels were not statistically different between the cirrhotic patients (with normal or altered glucose tolerance) and the control subjects, leading them to conclude that HbA1c is an unsatisfactory test for the diagnosis of altered glucose tolerance in patients with cirrhosis and that cirrhosis itself represents a risk factor for the development of alterations in glucose metabolism. Therefore, routine oral glucose tolerance testing is warranted in these patients [12]. Nomura et al. compared HbA1c levels and other measures of blood glucose control in a cirrhosis (LC) and healthy control (N) group with patients with diabetes mellitus (DM) or patients with chronic hepatitis $(\mathrm{CH})$ without evidence of cirrhosis [13]. HbA1c levels and the mean values of FPG for the past month were compared and were as follows: LC group, $6.40 \pm 0.36 \%$ (mean \pm standard error of the mean) and $130 \pm 20 \mathrm{mg} / \mathrm{dL}$, respectively; $\mathrm{DM}$ group, $10.29 \pm 0.45 \%$ and $172 \pm 11 \mathrm{mg} / \mathrm{dL}$, respectively; $\mathrm{CH}$ group, $10.70 \pm 0.86 \%$ and $176 \pm 21 \mathrm{mg} / \mathrm{dL}$, respectively; $\mathrm{N}$ group, $6.52 \pm 0.11 \%$ and $83 \pm 1 \mathrm{mg} / \mathrm{dL}$, respectively. The important finding in the study was that HbA1c in the LC group was similar to that in the $\mathrm{N}$ group, although the FPG in the former was higher $(p<0.05)$. HbA1c in the LC group was lower than that in DM or $\mathrm{CH}$ groups in spite of these patients having equivalent glucose intolerance. These results led the authors to suggest caution in the interpretation of HbA1c levels in hepatic cirrhosis [13]. Trenti et al. compared serum fructosamine and HbA1c levels in 98 cirrhotic patients with those of normal controls [15]. The mean HbA1c values of cirrhotic patients with normal glycemic control were significantly lower than normal, and only a few cirrhotic patients with diabetes showed high values of HbA1c, indicating that HbA1c is often underestimated in these patients. To the contrary, serum fructosamine levels were on average higher than normal in nondiabetic patients, but they were significantly higher in diabetic patients than in nondiabetics. The authors concluded that in diabetic patients with liver cirrhosis fructosamine seems to be a more suitable test than $\mathrm{HbA1c}$ for monitoring blood glucose levels [15]. Orsi et al. [10], reviewed all studies pertaining to the diagnosis of diabetes in patients with cirrhosis. In their review, two studies diagnosed diabetes on the basis of HbA1c, but none compared its utility against OGTT $[17,18]$. In our study, the direct comparison of HbA1c values with those of previous studies was difficult, but these previous data strongly indicated that $\mathrm{HbA1c}$ may not be an appropriate measure for the diagnosis or monitoring of patients with diabetes.

The prevalence of anemia was $90 \%$ in our study population. The presence of moderate to severe degree of anemia affected the performance of HbA1c in our study as described in 
"Results". In a recent systematic review, English et al. found that HbA1c is likely to be affected by iron deficiency anemia (IDA), possibly resulting in a spurious increase in HbA1c values and that non-IDA may lead to decreased HbA1c values [24]. Overall only two studies (both in non-CLD subjects) were identified in their systematic review which analyzed the prevalence of diabetes by OGTT-based criteria and HbA1cbased criteria taking anemia into account $[17,18]$. The current study therefore adds significantly to the knowledge gap in this area. In a study was from India, Hardikar et al. [25] investigated a population of young Indian subjects, among whom $30.8 \%$ had vitamin $\mathrm{B} 12$ deficiency, $15 \%$ had folate deficiency and 30\% had multiple nutrient deficiencies. In the multivariate analysis, B12 and folate were not significantly related to HbA1c levels. The authors postulated that where mixed nutrient deficiencies are observed, the combination of pathologies will lead to variable and unpredictable effects on HbA1c levels depending on the relative degree of each nutrient deficiency [25].

The prevalence of diabetes was 35.4 and $34.3 \%$ in outpatients and inpatients, respectively, indicating that hospitalized patients with CLD can undergo OGTT when they are stable and ready for discharge. Stress hyperglycemia may not significantly alter the results in such patients.

The prevalence of moderate to severe anemia was $53.8 \%$ in the subjects from the OPD and $63.6 \%$ in the hospitalized patients. This may be one of the reasons underlying the better performance of $\mathrm{HbA1c}$ in OPD subjects, as discussed in the "Results". We did not find any significant differences between different degrees of severity of liver disease.

Our study has its strengths and limitations. The major strength of the study is that it adds new information in multiple domains. The evaluation of HbA1c as compared to OGTT taking into account the degree of anemia has been evaluated for the first time. Since there are specific advantages to HbA1c testing, this study adds vital piece of information on its utility in patients with cirrhosis. One of the major limitations of our study is that is was a single-center study with a sample size of 100 patients. Due to this small sample size, the effect of different etiologies of cirrhosis and different etiologies of anemia could not be evaluated. The effect of gender and the effect of different complications also could not be evaluated. The results need to be substantiated in a larger study.

Future studies should try to address the above limitations. An aspect which also needs to be explored in future studies is the incidence of diabetes and predictive value of $\mathrm{HbA} 1 \mathrm{c}$ for future diabetes in patients with cirrhosis.

To summarize, taking OGTT as the gold standard, we found that the sensitivity of HbA1c was good when used in outpatients with cirrhosis. We noted that the sensitivity decreased when HbA1c was used for hospitalized patients, suggesting that it is not a good test for diagnosis of diabetes in such cases. It also performed poorly if the patient had moderate to severe anemia.

\section{ACKNOWLEDGEMENTS}

Funding. This study was supported by Indian Council of Medical Research-Short Term Studentship [2015-01083] 2015 awarded to Tejasav Sehrawat. The funding agency played no role in designing the study, in the collection, analysis and interpretation of data, in the writing of the report and in the decision to submit the article for publication.

Authorship. All named authors meet the International Committee of Medical Journal Editors (ICMJE) criteria for authorship for this article, take responsibility for the integrity of the work as a whole, and have given their approval for this version to be published.

Disclosures. Tejasav Sehrawat, Anuraag Jindal, Paaras Kohli, Amit Thour, Jasbinder Kaur Atul Sachdev and Yashdeep Gupta have nothing to disclose.

Compliance with Ethics Guidelines. All procedures performed were in accordance with the ethical standards of the institutional 
research committee and with the 1964 Helsinki declaration and its later amendments or comparable ethical standards. Informed consent was obtained from all individual participants included in the study. Ethics committee approval was taken for this study.

Open Access. This article is distributed under the terms of the Creative Commons Attribution-NonCommercial 4.0 International License (http://creativecommons.org/licenses/ by-nc/4.0/), which permits any noncommercial use, distribution, and reproduction in any medium, provided you give appropriate credit to the original author(s) and the source, provide a link to the Creative Commons license, and indicate if changes were made.

\section{REFERENCES}

1. Suk KT, Baik SK, Yoon JH, et al. Revision and update on clinical practice guideline for liver cirrhosis. Korean J Hepatol. 2012;18:1-21.

2. Tsochatzis EA, Bosch J, Burroughs AK. Liver cirrhosis. Lancet. 2014;383:1749-61.

3. Lozano R, Naghavi M, Foreman K, et al. Global and regional mortality from 235 causes of death for 20 age groups in 1990 and 2010: a systematic analysis for the Global Burden of Disease Study 2010. Lancet. 2012;380:2095-128.

4. Elkrief L, Rautou PE, Sarin S, et al. Diabetes mellitus in patients with cirrhosis: clinical implications and management. Liver Int. 2016;36:936-48.

5. Tuomi T, Santoro N, Caprio S, et al. The many faces of diabetes: a disease with increasing heterogeneity. Lancet. 2014;383:1084-94.

6. American Diabetes Association. Classification and diagnosis of diabetes: standards of medical care in diabetes-2018. Diabetes Care. 2018;41(Suppl 1):S13-27.

7. Zheng Y, Ley SH, Hu FB. Global aetiology and epidemiology of type 2 diabetes mellitus and its complications. Nat Rev Endocrinol. https://doi.org/10. 1038/nrendo.2017.151.

8. GBD 2013 Mortality and Causes of Death Collaborators. Global, regional, and national age-sex specific all-cause and cause-specific mortality for 240 causes of death, 1990-2013: a systematic analysis for the Global Burden of Disease Study 2013. Lancet. 2015;385:117-71.

9. International Diabetes Federation. IDF Diabetes Atlas-7th edition. Diabetes Atlas http://www. diabetesatlas.org/ (2015).

10. Orsi E, Grancini V, Menini S, et al. Hepatogenous diabetes: is it time to separate it from type 2 diabetes? Liver Int. 2017;37:950-62.

11. Nadelson J, Satapathy SK, Nair S. Glycated hemoglobin levels in patients with decompensated cirrhosis. Int J Endocrinol 2016; article ID 8390210. https://doi.org/10.1155/2016/8390210

12. Cacciatore L, Cozzolino G, Giardina MG, et al. Abnormalities of glucose metabolism induced by liver cirrhosis and glycosylated hemoglobin levels in chronic liver disease. Diabetes Res. 1988;7:185-8.

13. Nomura Y, Nanjo K, Miyano M, et al. Hemoglobin A1 in cirrhosis of the liver. Diabetes Res. 1989;11:177-80.

14. Lahousen T, Hegenbarth $\mathrm{K}$, Ille R, et al. Determination of glycated hemoglobin in patients with advanced liver disease. World J Gastroenterol. 2004;10:2284-6.

15. Trenti T, Cristani A, Cioni G, et al. Fructosamine and glycated hemoglobin as indices of glycemic control in patients with liver cirrhosis. Int J Clin Lab Res. 1990;20:261-7.

16. Shetty A, Wilson S, Kuo P, et al. Liver transplantation improves cirrhosis-associated impaired oral glucose tolerance. Transplantation. 2000;69:2451-4.

17. Lunati ME, Grancini V, Agnelli F, et al. Metabolic syndrome after liver transplantation: short-term prevalence and pre- and post-operative risk factors. Dig Liver Dis. 2013;45:833-9.

18. Grancini V, Trombetta M, Lunati ME, et al. Contribution of $\beta$-cell dysfunction and insulin resistance to cirrhosis-associated diabetes: role of severity of liver disease. J Hepatol. 2015;63:1484-90.

19. Peng Y, Qi X, Guo X. Child-Pugh versus MELD score for the assessment of prognosis in liver cirrhosis: a systematic review and meta-analysis of observational studies. Medicine (Baltimore). 2016;95:e2877.

20. World Health Organization. Haemoglobin concentrations for the diagnosis of anaemia and assessment of severity. http://www.who.int/vmnis/ indicators/haemoglobin/en/ Accessed 8 Aug 2017. 
21. World Health Organization. Department of Noncommunicable Disease Surveillance. Definition, diagnosis and classification of diabetes mellitus and its complications: report of a WHO consultation. Part 1, Diagnosis and classification of diabetes mellitus 1999. http://apps.who.int/iris/handle/ 10665/66040. Accessed 16 Dec

22. Anjana RM, Pradeepa R, Deepa M, et al. Prevalence of diabetes and prediabetes (impaired fasting glucose and/or impaired glucose tolerance) in urban and rural India: phase I results of the Indian Council of Medical Research-INdia DIABetes (ICMR-INDIAB) study. Diabetologia. 2011;54:3022-7.
23. Gonzalez-Casas R, Jones EA, Moreno-Otero R. Spectrum of anemia associated with chronic liver disease. World J Gastroenterol. 2009;15:4653-8.

24. English E, Idris I, Smith G, et al. The effect of anaemia and abnormalities of erythrocyte indices on HbA1c analysis: a systematic review. Diabetologia. 2015;58:1409-21.

25. Hardikar PS, Joshi SM, Bhat DS, et al. Spuriously high prevalence of prediabetes diagnosed by $\mathrm{HbA}(1 \mathrm{c})$ in young Indians partly explained by haematological factors and iron deficiency anemia. Diabetes Care. 2012;35:797-802. 